\title{
Brief psychological interventions for psychiatric disorders in young people with long term physical health conditions: a systematic review and meta-analysis
}

M Catanzano, S Bennett, PhD, C Sanderson, M Patel, G Manzotti, E Kerry, A Coughtrey, PhD, H Liang, I Heyman, PhD, R Shafran, PhD

Authors' affiliations: UCL Great Ormond Street Institute of Child Health, London, UK.

Funding: this review was carried out as part of a PhD funded by The Beryl Alexander Charity and Great Ormond Street Hospital Children's Charity.

Corresponding Author: Matteo Catanzano

Name: Matteo Catanzano

Address: $4^{\text {th }}$ floor - WTB, Population Policy Practice, UCL GOS Institute of Child Health, 30 Guilford street, London, WC1N 1EH, UK

Email: matteo.catanzano@nhs.net

Tel: $+44(0) 2074059200$

Key words: brief; child and adolescent; chronic illness; long term condition; mental health; psychological intervention. 


\section{Introduction}

Rates of psychiatric disorders such as depression (Pinquart \& Shen, 2011c), disruptive behaviour disorders (i.e. oppositional and conduct disorders) (Pinquart \& Shen, 2011b), post-traumatic stress (Pinquart, 2018), anxiety (Pinquart \& Shen, 2011a) and eating disorders (Conviser, Fisher, \& McColley, 2018) are significantly elevated in young people with long term physical health conditions (Almane et al., 2019; Chun et al., 2015; Khanna et al., 2019; Quilter, Hiraki, \& Korczak, 2019). This is a public health concern as large numbers of children and young people have long term physical health conditions, with prevalence rates of up to $23 \%$ in the UK (Brooks, Magnusson, Klemera, Spencer, \& Morgan, 2011) and 25\% in the US (Van Cleave, Gortmaker, \& Perrin, 2010; van der Lee, Mokkink, Grootenhuis, Heymans, \& Offringa, 2007). Untreated co-morbid psychiatric disorders in paediatric patients have been associated with more impaired physical functioning (Ding, Hall, Jacobs, \& David, 2008), lower quality of life (Johnson, Jones, Seidenberg, \& Hermann, 2004), sub-optimal disease management (Sildorf et al., 2018) and increased mortality during admission to hospital (Olusunmade, Qadir, Akyar, Farid, \& Aggarwal, 2019). The impact can extend to the whole family. Higher rates of parenting stress (Cousino \& Hazen, 2013) and emotional problems in siblings (Sharpe \& Rossiter, 2002) have been found in families of children with a long term physical condition compared to those without. There is evidence to suggest that this higher rate of emotional problems in siblings can persist in the long term (O'Neill \& Murray, 2016). There are also economic costs of co-morbidity, as those with comorbid psychiatric disorders in the context of chronic physical illness experience more frequent and longer admissions to hospital, which can be up to 5 times more expensive than those of their peers without a psychiatric co-morbidity (Zima et al., 2016). For frequency of hospital admissions, this is especially true in young people where the psychiatric comorbidity is a developmental disorder, attention deficit hyperactivity disorder or anxiety disorder (Zima et al., 2016). 
In the UK, psychiatric disorders in children and young people are treated within specialist Child and Adolescent Mental Health Services. Yet, accessibility of treatments in the community appears limited. For example, for $78 \%$ of young people referred to Child and Adolescent Mental Health Services with problems in addition to their long term physical health condition, the outcome of the referral was not known (Children's Commissioner, 2016). Even for the few who access treatment, research in this area suggests that interventions received are patchy, inconsistent and not always compliant with NICE guidelines (Welch, Shafran, Heyman, Coughtrey, \& Bennett, 2018). For mild depression in children and young people, NICE recommend as first line treatment a psychological intervention such as digital cognitive-behavioural therapy (CBT), group CBT, group nondirective supportive therapy (NDST) or group interpersonal psychotherapy (IPT) for 8 - 12 sessions (NICE, 2019); for anxiety disorders, individual CBT of 8-12 sessions of 45 minutes' duration (for social anxiety) or group CBT (NICE, 2013b); for disruptive behaviour disorders (conduct disorder or ODD) (NICE, 2013a) and challenging behaviour in young people with an intellectual disability (NICE, 2015) parent training interventions. Poor access to evidencebased mental health support is not due to a lack of known effective treatments for young people (Weisz et al., 2017). Evidence suggests that cognitive behavioural therapy (CBT) treatments may be effective in young people with mental health needs in addition to long term physical health conditions. This has been explored for a range of conditions - for example epilepsy, inflammatory bowel disease, functional symptoms and chronic pain (Moore et al., 2019) and may require practical adaptations, such as more flexibility around appointments (Bennett, Shafran, Coughtrey, Walker, \& Heyman, 2015). Some psychological interventions included adaptations to content to take account of the long term physical health condition (for example in the PASCET-PI studies by Szigethy and colleagues, adaptations to content included information about inflammatory bowel disease, techniques for coping with abdominal pain, identifying negative cognitions about inflammatory bowel disease), but at present there is insufficient evidence to know whether these are required or improve efficacy (Moore et al., 2019). There was insufficient evidence to assess whether these interventions 
are cost-effective (Moore et al., 2019). It is likely that barriers to accessing care, although compounded by a long term physical health condition, are similar to those of young people without a long term physical health condition. These include a lack of trained therapists, costs, logistical issues like child care, transport, work demands and time (Owens et al., 2002).

A promising approach to increase access to evidence-based therapy for adults with comorbid long-term conditions and anxiety/depression (Kellett et al., 2016), involves using 'brief' CBT interventions as part of stepped care. This model has been extended to young people with anxiety/depression/disruptive behaviour in the absence of a co-morbid long term physical health condition, with similar results (Edbrooke-Childs, Calderon, Wolpert, \& Fonagy, 2015). Although the definitions of 'brief' vary, they usually involve $\leq 10$ sessions with $\leq 6$ hours of therapist contact per patient often include self-help materials, and can be delivered by mental health workers with less specialist training (Cape \& Kendall, 2011; Stallard, 2017). This is in contrast to traditional 'high-intensity' therapies that typically involve 12-16 hour-long sessions, by a highly trained mental health professional.

Brief interventions have the potential to reach more people. The mechanisms may include a reduction in time spent travelling to clinic for patients, travel costs, time off work or school (Stallard, 2017). These benefits are likely to be particularly important to families who already have to attend a number of appointments for their child's physical illness. Moreover, they allow mental health workers with less specialist training to deliver interventions with similar outcomes at a reduced cost to society (Creswell et al., 2017), thereby increasing capacity and as a result reducing waiting times.

In order to determine what brief evidence-based psychological treatments for paediatric hospital patients are effective, a review of the evidence is needed. There have been reviews in children and adolescents of specific forms of brief CBT in specific long term physical 
health conditions, such as e-health interventions for anxiety and depression (Thabrew et al., 2017), or remotely delivered interventions for chronic pain/headache (Fisher, Law, Palermo, \& Eccleston, 2015). A subgroup analysis of a broader review of psychological interventions for young people with long term physical health conditions looked at "brief" therapy, defined as $<6$ sessions or $<6$ hours, rather than 'brief' as defined above. In this particular subgroup analysis, Thabrew and colleagues synthesised different types of psychological therapies in the same meta-analysis (e.g. CBT and family therapy) (Thabrew et al., 2018), which precluded examining differential efficacy of individual modalities, as in reviews of high intensity interventions for young people with long term physical health conditions these have differed (Moore et al., 2019). Finally, this was done two years ago in a fast moving field and important additional papers have been published since then.

To our knowledge none have reviewed brief interventions (including guided self-help) in long term physical health conditions in young people with elevated psychiatric symptoms as a whole.

\section{Objectives}

This systematic review aimed to explore:

- the efficacy of brief interventions targeting elevated symptoms of psychiatric disorders for young people with long term physical health conditions versus any comparator.

- the effects of such interventions on other key aspects of individual functioning (e.g. quality of life or physical health).

- factors that may moderate the efficacy or acceptability of these interventions.

\section{Methods}

Protocol pre-registration and outcome reporting 
The review protocol was registered to PROSPERO on the 22/03/2019 (PROSPERO ID: CRD42019121299). Outcomes were reported in accordance with the Preferred Reporting Items for Systematic Reviews and Meta-Analyses (PRISMA) Statement (Moher, Liberati, Tetzlaff, Altman, \& Group, 2009).

Criteria for considering studies for this review Inclusion criteria

Population

- Studied participants up to 18 years of age.

- Participants either had a psychiatric disorder or elevated symptoms of a psychiatric disorder at baseline (defined as the mean of the sample in each arm being above an accepted cut off for a validated mental health measure).

- Participants all diagnosed with a long term physical health condition.

- We defined long term physical health conditions as lasting for at least 3 months, causing functional impairment, necessitating medical care and where cure is considered unlikely. This definition was in line with that of a recent evidence synthesis on the topic (Moore et al., 2019) . Moore and colleagues (2019) report defining long term physical health conditions, by drawing on a systematic review of definitions of chronic health conditions in childhood (van der Lee et al., 2007) and selecting a combination of those most frequently cited. Conditions included in the search: AIDS and HIV, asthma, cancer, chronic pain, cleft palate, cystic fibrosis, deafness/hearing impairment, diabetes, epilepsy, headache, heart disease, inflammatory bowel disease (IBD), kidney disease, liver disease, migraine, sickle cell anaemia, spina bifida and visual impairment. 
- Studied a brief intervention defined as: interventions involving $\leq 10$ sessions with $\leq 6$ hours of therapist contact per patient (Cape \& Kendall, 2011; Stallard, 2017). In the case of group treatments, we multiplied the total number of hours of the group by the number of therapists and then divided it by the average number of patients in the group (Rodgers et al., 2012).

- This included all brief interventions with a cognitive and/or behavioural component (e.g. CBT, parenting interventions), relational/insight-oriented component (family/systemic therapy, psychodynamic therapy), as well as interventions using physiological, sensory and/or complementary approaches (e.g. music therapy, massage therapy, biofeedback, hypnosis) to target symptoms of psychiatric disorders and reduce distress/impairment.

\section{Comparator}

- Any control group (e.g. treatment as usual, waitlist control, other psychological therapy, pharmacological therapy or other).

\section{Outcomes}

- Reported a child-related mental health measure (the measure had to relate to the mental health of the child and not the parent/carer, although parent-reports of child health/behaviour were acceptable) at post-intervention and/or follow-up (any length).

- Examples of outcome measures include: symptoms of depression (e.g. Beck Depression Inventory for Youth), anxiety (e.g. State-Trait Anxiety Inventory for Children) behavioural disorders (e.g. Child Behaviour Checklist). 
- Randomised controlled trials (RCTs) only.

\section{Exclusion criteria}

We excluded studies that were not in a language that was known to the reviewers (i.e. studies not in Italian, French or English).

\section{Search Methods}

Electronic Searches, citation searches, reference list searches and grey literature searches were independently undertaken by MC and MP (a psychological wellbeing practitioner).

\section{Electronic searches}

EMBASE, MEDLINE, PsycINFO databases were searched from inception to 12/02/2020. Grey/unpublished literature was also included, through searches of PsycExtra, Google and Google Scholar. Broadly, the search terms were categorised into 5 primary areas; (1) Children and young people, (2) Brief psychological interventions (3) Psychiatric disorders (4) Chronic illness 5) RCT. Please see supplementary materials for the full electronic search strategy.

\section{Other searches}

Additional literature was found through reviewing the reference lists of relevant systematic reviews in the area.

\section{Data collection and analysis}

Study selection

Study selection was performed independently by two reviewers, MC and MP. The kappa agreement for included/excluded studies at the full-text article screening stage was 0.879 
$(\mathrm{P}<0.001$; substantial agreement). Where disagreements arose this was resolved through discussion with SB.

\section{Data extraction and management}

A data extraction form was developed and adapted from the Cochrane handbook template (Higgins et al., 2019). Data on study details and aims, participants, mental health measures at baseline, intervention, number of sessions, and duration of sessions, outcome measures, findings and study quality were extracted independently by two reviewers, MC and CS (a clinical psychologist). The kappa agreement for extraction of the primary mental health outcome measure between the two independent reviewers was $0.833(p<0.001$; substantial agreement). Where disagreements arose this was resolved through discussion with SB.

\section{Assessment of risk of bias in included studies}

Risk of bias was independently assessed by two reviewers (MC and CS) using the Cochrane Risk of Bias Tool (Higgins et al., 2011). This assesses allocation concealment, sequence generation, blinding, selective outcome reporting and incomplete outcome reporting. The kappa agreement for risk of bias ratings between the two independent reviewers was 0.648 ( $p<0.001$; substantial agreement). Studies with no obvious evidence of selective reporting required a protocol to cross-check this, otherwise they were coded as 'unclear risk of bias' in that domain. Studies where only self-report measures were used, were coded as unclear risk of bias, unless a blind assessor was used, in which case they were coded as low risk of bias. Where disagreements arose this was resolved through discussion with SB.

\section{Data synthesis}

The meta-analysis was carried out using Review Manager 5 (RevMan), according to the Cochrane guidelines (Higgins et al., 2019). For each relevant outcome, post-intervention mean, standard deviation and sample size were extracted. Comparisons of clinical efficacy 
were summarised with the standardised mean difference (Hedges' g) between intervention and control group post-intervention as studies assessed similar outcomes but measured them in a variety of ways (e.g. different self-report questionnaires for anxiety symptoms) (Higgins et al., 2019). Hedges' g was used to calculate effect size at post-intervention, with 95\% confidence intervals (Cls) and p-values. A meta-analysis was considered feasible where multiple studies examined the same type of intervention (e.g. CBT) and used the same outcome category (e.g. anxiety measure). A random-effects model was used to pool effect sizes, due to no two studies addressing the research question using the same methodology. When a quantitative synthesis was not possible, due to insufficient suitable studies for a meta-analysis or where certain outcomes were not reported (e.g. standard deviation), these were synthesised narratively.

We contacted authors for apparent missing data. As this was unsuccessful, we reported missing data as 'not reported' in Tables 1 and 2. Heterogeneity was calculated using Tau² and $\mathrm{I}^{2}$. As per the Cochrane guidelines, $\mathrm{I}^{2}$ was roughly interpreted as follows:

- $0 \%$ to $40 \%$ : might not be important;

- $30 \%$ to $60 \%$ : may represent moderate heterogeneity;

- $50 \%$ to $90 \%$ : may represent substantial heterogeneity;

- $75 \%$ to $100 \%$ : considerable heterogeneity.

All meta-analysis, forest plots and related calculations were done using Review Manager 5.3 (Revman version 5.3). Assessment of Publication bias was planned by examining funnel plots for asymmetry in Revman 5.3, but this was not possible due to the limited number of studies with similar characteristics entered in the meta-analysis. For the same reason regression-based assessments of publications bias were unable to be used.

Summary of findings 
The GRADE system (Schünemann, Brożek, Guyatt, \& Oxman, 2013) was used to assess quality of the body of evidence, using GRADEpro (version 3.6). Four categories are used in this approach (high, moderate, low and very low) to rate the quality of the evidence available. All RCTs start at a high level. Evidence from RCTs can be downgraded if there are indications of low quality such as high risk of bias or small sample sizes. Where this occurred it was noted in the footnotes of the 'Summary of findings' table (Table 4).

\section{Results}

Description of studies

The initial search identified 944 papers after duplicates had been removed (see PRISMA flow diagram, Figure 1). A total of 12 studies (with 425 children and adolescents) were found to meet the inclusion criteria of the review (Bennett, 2017; Bufalini, 2009; Field et al., 1998; Freedenberg, Hinds, \& Friedmann, 2017; Hains, 2000; Hickman, Jacobson, \& Melnyk, 2015; Jastrowski Mano et al., 2013; Liossi \& Hatira, 1999; Scharff, Marcus, \& Masek, 2002; Sharma, Mehta, \& Sagar, 2017; Yetwin, 2011; Zhang, Mo, Torres, \& Huang, 2019). Study characteristics were summarised in Table 1. 


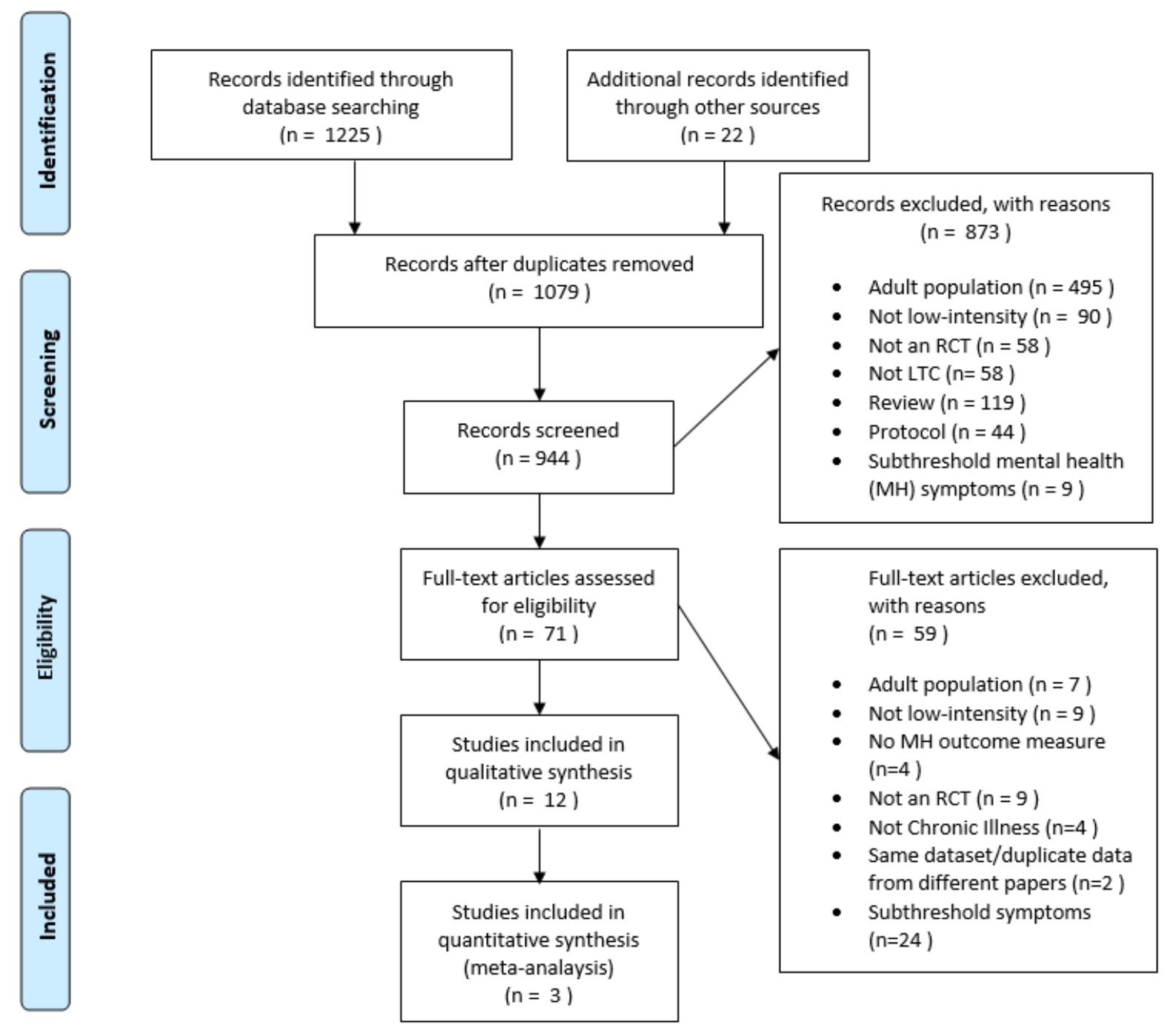

Figure 1. PRISMA flow diagram of included studies in the review 
Table 1. Characteristics of included studies

\begin{tabular}{|c|c|c|c|c|c|c|c|c|c|c|c|c|c|c|c|}
\hline Study & $\begin{array}{l}\text { Participant } \\
\text { n (\% } \\
\text { female) }\end{array}$ & $\begin{array}{l}\text { Age } \\
\text { of } \\
\text { Participants } \\
\text { M years (SD) }\end{array}$ & $\begin{array}{l}\text { Psychiatric } \\
\text { disorder }\end{array}$ & $\begin{array}{l}\text { Long Term } \\
\text { Condition }\end{array}$ & Intervention & $\begin{array}{l}\text { Intervention } \\
\text { provider }\end{array}$ & $\begin{array}{l}\text { Intervention } \\
\text { structure }\end{array}$ & $\begin{array}{l}\text { Dose } \\
\text { (hours) }\end{array}$ & $\begin{array}{l}\text { Intervention } \\
\text { recipient }\end{array}$ & $\begin{array}{l}\text { Delivery } \\
\text { method }\end{array}$ & $\begin{array}{l}\text { Delivery } \\
\text { format }\end{array}$ & Comparator & Site & $\begin{array}{l}\text { Time } \\
\text { points }\end{array}$ & Country \\
\hline $\begin{array}{l}\text { Bennett } \\
\text { (2017) }\end{array}$ & $34(50 \%)$ & 11.28 (3.29) & $\begin{array}{l}\text { Anxiety, } \\
\text { depression } \\
\text { and } \\
\text { disruptive } \\
\text { behavior }\end{array}$ & $\begin{array}{l}\text { Neurological } \\
\text { conditions } \\
(88 \% \\
\text { epilepsy) }\end{array}$ & $\begin{array}{l}\text { Cognitive } \\
\text { Behavioural } \\
\text { Therapy guided } \\
\text { self-help }\end{array}$ & $\begin{array}{l}\text { Trained } \\
\text { research } \\
\text { assistants } \\
\text { supervised } \\
\text { by clinical } \\
\text { psychologist } \\
\text { (who also } \\
\text { took on } \\
\text { cases) }\end{array}$ & $\begin{array}{l}\text { Up to } 10 \\
\text { sessions of } \\
30 \text { minutes (total } \\
5 \text { hours max.) }\end{array}$ & $\begin{array}{l}5 \text { hours } \\
(\max )\end{array}$ & $\begin{array}{l}\text { Parent and/or } \\
\text { child }\end{array}$ & Phone & Individual & Waitlist control & Hospital & $\begin{array}{l}\text { Pre-, post- and } \\
\text { 3-months }\end{array}$ & UK \\
\hline $\begin{array}{l}\text { Bufalini et. } \\
\text { al. (2009) }\end{array}$ & $39(39 \%)$ & $\begin{array}{l}6.7 \text { (SD not } \\
\text { reported) }\end{array}$ & $\begin{array}{l}\text { Procedural } \\
\text { anxiety }\end{array}$ & Cancer & $\begin{array}{l}\text { Interactive } \\
\text { Music therapy }\end{array}$ & $\begin{array}{l}\text { Doctor } \\
\text { trained in } \\
\text { music } \\
\text { therapy }\end{array}$ & $\begin{array}{l}15 \text { min prior to } \\
\text { procedure and } \\
\text { up to sedation. } \\
\text { (total time not } \\
\text { reported) }\end{array}$ & $\begin{array}{l}\text { Not } \\
\text { reported }\end{array}$ & Child & f2f & Individual & $\begin{array}{l}\text { Treatment as usual } \\
\text { (usual sedation) }\end{array}$ & Hospital & $\begin{array}{l}\mathrm{T} 1 ; \mathrm{T} 2 ; \mathrm{T} 3 ; \mathrm{T} 4 \\
\text { (4 time points } \\
\text { from beginning } \\
\text { to end of } \\
\text { intervention) }\end{array}$ & Italy \\
\hline $\begin{array}{l}\text { Field et. al. } \\
\text { (1998) }\end{array}$ & $32(38 \%)$ & $\begin{array}{l}9.15(S D \text { not } \\
\text { reported ; } \\
\text { range: } 4-14)\end{array}$ & Anxiety & Asthma & $\begin{array}{l}\text { Massage } \\
\text { therapy }\end{array}$ & $\begin{array}{l}\text { Massage } \\
\text { therapist } \\
\text { taught parent } \\
\text { of child to } \\
\text { administer } \\
\text { massages }\end{array}$ & $\begin{array}{l}\text { One-off } \\
\text { demonstration, } \\
\text { written } \\
\text { instructions and } \\
\text { a videotaped } \\
\text { demo were given } \\
\text { to parents who } \\
\text { then massaged } \\
\text { child } 30 \text { mins a } \\
\text { night for } 30 \text { days } \\
\text { (total time not } \\
\text { reported) }\end{array}$ & $\begin{array}{l}\text { Not } \\
\text { reported }\end{array}$ & Child & $\mathrm{f} 2 \mathrm{f}$ & Individual & $\begin{array}{l}\text { Progressive Muscle } \\
\text { Relaxation }\end{array}$ & Hospital & Pre- and post- & USA \\
\hline $\begin{array}{l}\text { Hains et. al. } \\
(2000)\end{array}$ & $15(53 \%)$ & $\begin{array}{l}\text { Not reported } \\
\text { (range:12-15) }\end{array}$ & Anxiety & $\begin{array}{l}\text { Type I } \\
\text { Diabetes }\end{array}$ & $\begin{array}{l}\text { Group } \\
\text { Cognitive } \\
\text { Behavioural } \\
\text { Therapy }\end{array}$ & $\begin{array}{l}2 x \\
\text { Psychology } \\
\text { PhD }\end{array}$ & $\begin{array}{l}6 \times 1 \text { hour } \\
\text { sessions per } \\
\text { group of } 4 \\
\text { youths }\end{array}$ & 1h30mins & Child & f2f & Group & Waitlist control & Hospital & $\begin{array}{l}\text { Pre-, post- and } \\
1 \text { month }\end{array}$ & USA \\
\hline $\begin{array}{l}\text { Hickman et. } \\
\text { al. (2015) }\end{array}$ & $32(72 \%)$ & $\begin{array}{l}\text { Intervention } \\
\text { group: } 15.38 \\
(0.96) \\
\text { Control: } \\
14.8(1.17)\end{array}$ & Depression & Headaches & $\begin{array}{l}\text { Cognitive } \\
\text { behavioural } \\
\text { skills building }\end{array}$ & $\begin{array}{l}\text { Neurology } \\
\text { Nurse } \\
\text { trained in } \\
\text { Cognitive } \\
\text { behavioural } \\
\text { skills building }\end{array}$ & $\begin{array}{l}\text { three office } \\
\text { sessions of } 30 \\
\text { minutes and four } \\
\text { telephone } \\
\text { sessions of } 20 \\
\text { minutes over } \\
\text { seven weeks } \\
\text { (total 2h50mins) }\end{array}$ & 2h50mins & Child & $\begin{array}{l}\text { Blended } \\
\text { phone/t2f }\end{array}$ & Individual & $\begin{array}{l}\text { Headache } \\
\text { education program }\end{array}$ & Hospital & Pre- and post- & USA \\
\hline
\end{tabular}




\begin{tabular}{|c|c|c|c|c|c|c|c|c|c|c|c|c|c|c|c|}
\hline $\begin{array}{l}\text { Freedenberg } \\
\text { et. al. (2017) }\end{array}$ & $46(63 \%)$ & $\begin{array}{l}\text { MBSR group: } \\
15.1(1.8) \\
\text { Video group: } \\
14.5(1.6)\end{array}$ & Anxiety & $\begin{array}{l}\text { Congenital } \\
\text { Heart } \\
\text { Disease }\end{array}$ & $\begin{array}{l}\text { Mindfulness- } \\
\text { Based Stress } \\
\text { Reduction }\end{array}$ & $\begin{array}{l}\text { Nurse } \\
\text { trained in } \\
\text { MBSR }\end{array}$ & $\begin{array}{l}6 \times 1.5 \text { hour } \\
\text { group sessions } \\
\text { (7-10 per group) }\end{array}$ & $1 \mathrm{~h}$ & Child & $\mathrm{f} 2 \mathrm{f}$ & Group & $\begin{array}{l}\text { Online video } \\
\text { support group }\end{array}$ & Hospital & $\begin{array}{l}\text { Pre-, post- and } \\
6 \text { months }\end{array}$ & USA \\
\hline $\begin{array}{l}\text { Jastrowski } \\
\text { et. al. } \\
\text { (2013) }\end{array}$ & $6(83 \%)$ & $\begin{array}{l}\text { Intervention } \\
\text { group: } 15 \\
(2.16) \\
\text { Control: } 12.5 \\
(0.71)\end{array}$ & Anxiety & Chronic pain & $\begin{array}{l}\text { Mindfulness- } \\
\text { Based Stress } \\
\text { Reduction }\end{array}$ & $\begin{array}{l}\text { MBSR } \\
\text { practitioner }\end{array}$ & $\begin{array}{l}6 \times 90 \text { minutes } \\
\text { group sessions } \\
\text { (4 per group) }\end{array}$ & 2h25mins & Child & $\mathrm{f} 2 \mathrm{f}$ & Group & $\begin{array}{l}\text { Psychoeducation } \\
\text { group }\end{array}$ & Hospital & $\begin{array}{l}\text { Pre-, post-, } 1 \\
\text { month and } 3 \\
\text { months }\end{array}$ & USA \\
\hline $\begin{array}{l}\text { Liossi et. al. } \\
\text { (1998) }\end{array}$ & $30(43 \%)$ & $8(2.5)$ & $\begin{array}{l}\text { Procedural } \\
\text { anxiety }\end{array}$ & Leukaemia & Hypnosis & $\begin{array}{l}\text { Clinical } \\
\text { Psychologist }\end{array}$ & $\begin{array}{l}2 \times 30 \text { minute } \\
\text { sessions }\end{array}$ & 1hour & $\begin{array}{l}\text { Child and } \\
\text { Parent }\end{array}$ & $\mathrm{f} 2 \mathrm{f}$ & Individual & CBT and control & Hospital & Pre- and post- & UK \\
\hline $\begin{array}{l}\text { Scharff et.al. } \\
\text { (1999) }\end{array}$ & $36(66 \%)$ & $\begin{array}{l}\text { Intervention } \\
\text { group: } 13.3 \\
(2.5) \\
\text { Handcooling } \\
\text { biofeedback } \\
\text { group: } 13.2 \\
(2.0) \\
\text { Control: } 12.0 \\
(2.7)\end{array}$ & Anxiety & Migraine & $\begin{array}{l}\text { Thermal } \\
\text { biofeedback } \\
\text { and cognitive } \\
\text { behavioural } \\
\text { stress } \\
\text { management } \\
\text { training }\end{array}$ & $\begin{array}{l}\text { PhD } \\
\text { psychologist }\end{array}$ & $\begin{array}{l}4 \times 1 \text { hour } \\
\text { sessions in } 6 \\
\text { weeks }\end{array}$ & 4 hours & Child & $\mathrm{f} 2 \mathrm{f}$ & Individual & $\begin{array}{l}\text { Handcooling } \\
\text { biofeedback and } \\
\text { Waitlist control }\end{array}$ & Hospital & $\begin{array}{l}\text { Pre-, post-, } \\
3 \text { months and } \\
6 \text { months }\end{array}$ & USA \\
\hline $\begin{array}{l}\text { Sharma et. } \\
\text { al. (2017) }\end{array}$ & $63(48 \%)$ & $13.90(2.60)$ & Anxiety & Headache & $\begin{array}{l}\text { Transdiagnostic } \\
\text { group Cognitive } \\
\text { Behavioural } \\
\text { Therapy }\end{array}$ & $\begin{array}{l}\text { Doctoral } \\
\text { level } \\
\text { students } \\
\text { used to } \\
\text { delivering } \\
\text { CBT, } \\
\text { supervised } \\
\text { by a clinical } \\
\text { psychologist } \\
\text { and } \\
\text { psychiatrist }\end{array}$ & $\begin{array}{l}12 \times 2 \text { hour group } \\
\text { sessions }(5-6 \\
\text { young people } \\
\text { per group) }\end{array}$ & $4 \mathrm{~h} 30 \mathrm{mins}$ & Child & $\mathrm{f} 2 \mathrm{f}$ & Group & $\begin{array}{l}\text { Treatment as usual } \\
\text { (pharmacotherapy) }\end{array}$ & Hospital & Pre- and post- & India \\
\hline $\begin{array}{l}\text { Yetwin } \\
\text { (2011) }\end{array}$ & $19(76 \%)$ & $\begin{array}{l}14.1 \\
(1.91)\end{array}$ & Anxiety & Chronic pain & $\begin{array}{l}\text { Heart rate } \\
\text { variability } \\
\text { Biofeedback }\end{array}$ & $\begin{array}{l}\text { PhD } \\
\text { psychologist }\end{array}$ & $\begin{array}{l}4 \text { treatment } \\
\text { sessions, lasting } \\
\text { 30-60mins }\end{array}$ & $\begin{array}{l}45 \operatorname{mins} \times 4 \\
=3 \text { hours }\end{array}$ & Child & $\mathrm{f} 2 \mathrm{f}$ & Individual & Waitlist control & Hospital & Pre- and post- & USA \\
\hline
\end{tabular}




\begin{tabular}{|c|c|c|c|c|c|c|c|c|c|c|c|c|c|c|c|}
\hline $\begin{array}{l}\text { Zhang et. al. } \\
\text { (2019) }\end{array}$ & $108(51 \%)$ & $\begin{array}{l}\text { NR } \\
\text { (range:8-18) }\end{array}$ & Anxiety & Cancer & $\begin{array}{l}\text { Cognitive } \\
\text { Behavioural } \\
\text { Therapy }\end{array}$ & $\begin{array}{l}\text { CBT } \\
\text { therapist }\end{array}$ & $\begin{array}{l}5 \text { sessions over } \\
5 \text { weeks (1 } \\
\text { assessment, } 3 \\
\text { treatment } \\
\text { sessions and } 1 \\
\text { to collect } \\
\text { questionnaires) }\end{array}$ & $\begin{array}{l}\text { Not } \\
\text { reported }\end{array}$ & $\begin{array}{l}\text { Child and } \\
\text { Parent }\end{array}$ & $\mathrm{f} 2 \mathrm{f}$ & Individual & Treatment as usual & Hospital & Pre- and post- & China \\
\hline
\end{tabular}


All studies involved different combinations of long term physical health conditions, psychiatric disorders and intervention types. Ten studies looked at anxiety, one at depression and one at 'emotional and behavioural' problems. The most common long term physical health conditions were cancer and headache. The majority of studies were delivered face-to-face and on an individual basis. Five studies were based on Cognitive Behavioural principles. Average therapist time per patient was 3 hours, in studies where this was reported. Two of the included studies were unpublished doctoral theses (Bennett, 2017; Yetwin, 2011).

\section{Risk of bias}

Risk of bias was assessed in accordance to Cochrane's 'risk of bias tool (Higgins et al., 2011) and summarised in Figures 2 and 3. Overall, there was evidence of risk of bias in all studies, due to difficulty in blinding participants and personnel. No studies had low risk of bias in all other domains. Interventions based on CBT principles tended to have a lower risk of bias. 


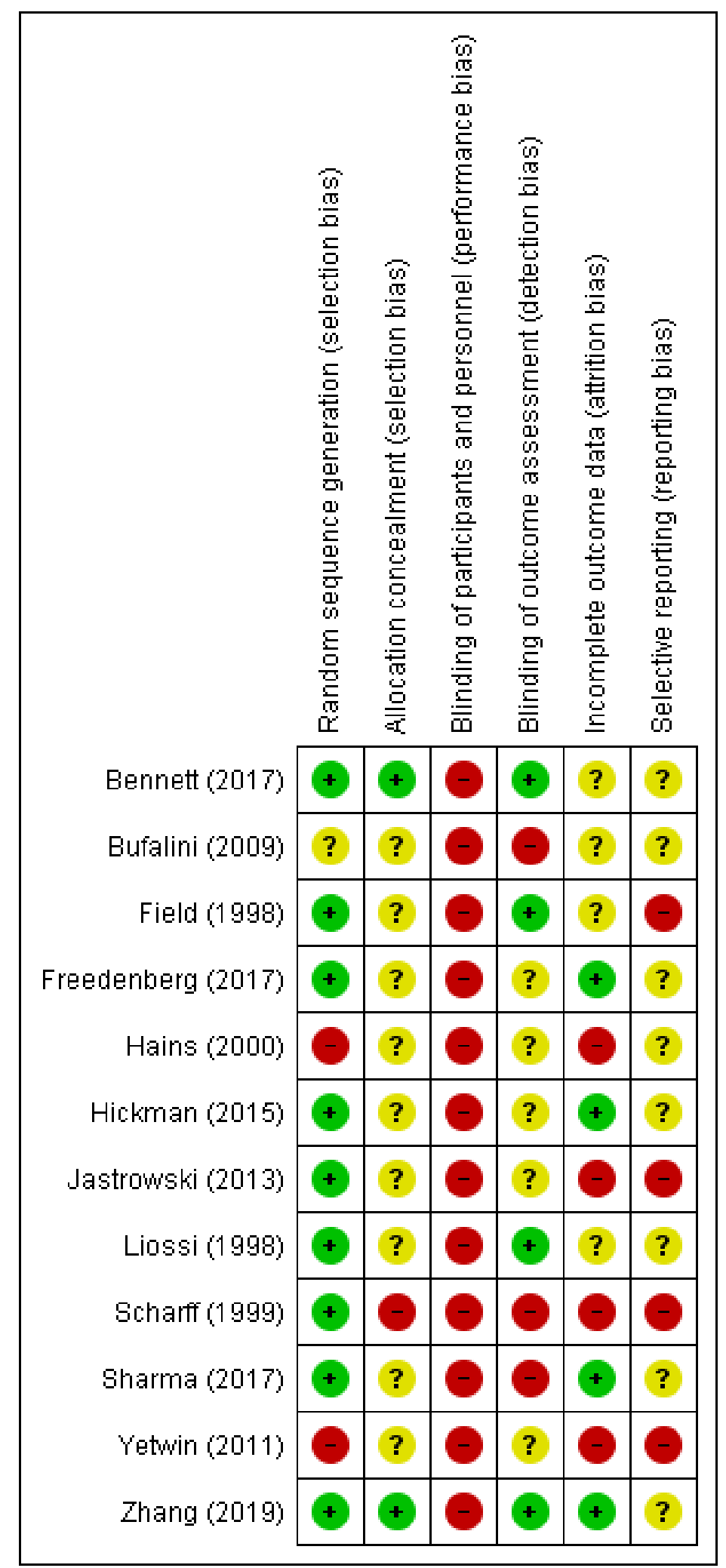

Figure 2. Risk of bias summary: review authors' judgements about each risk of bias item for each included study 


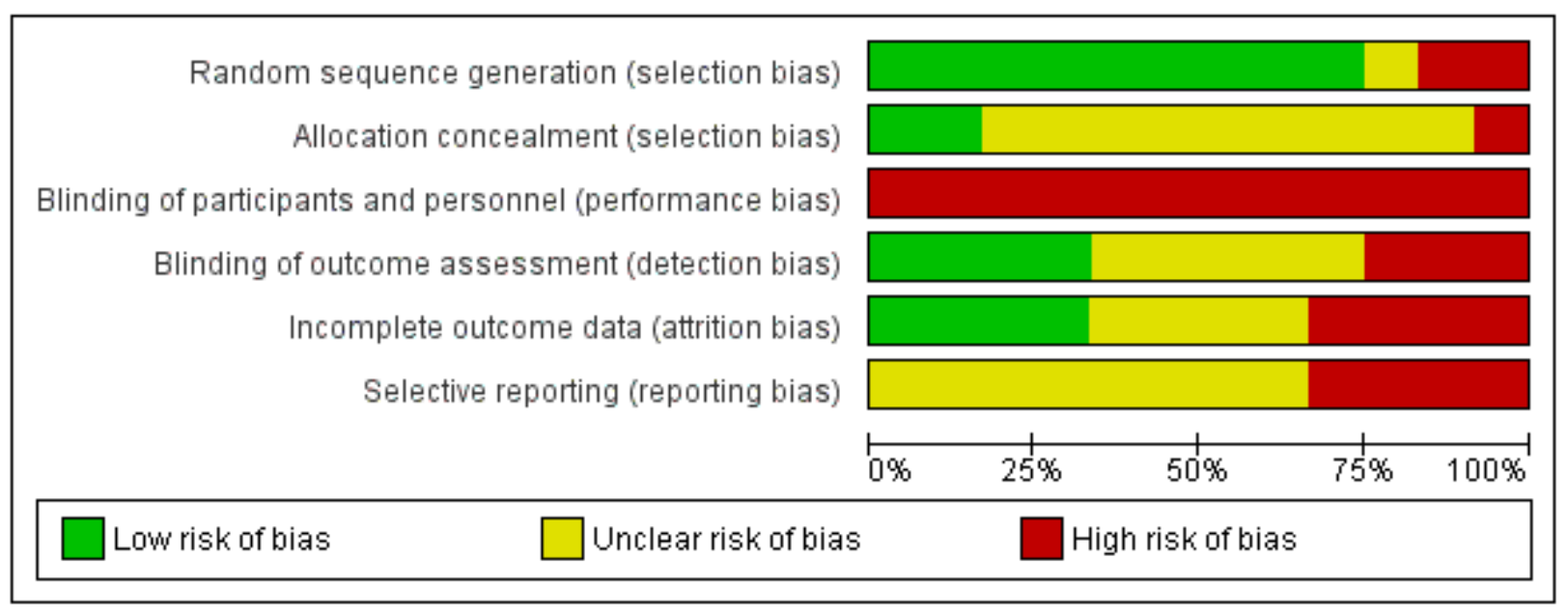

Figure 3. Risk of bias graph: review authors' judgements about each risk of bias item presented as percentages across all included studies 


\section{Efficacy}

Definitive conclusions cannot be drawn from the available evidence as most studies had small sample sizes and as a result were likely to be underpowered to detect statistically significant between group differences. Mental health outcomes of the included studies are summarised in Table 2. Other outcomes are summarised in Table 3. A summary of findings table for the main comparison: 'Brief interventions compared to any comparator for psychiatric disorders in children with long term physical health conditions is presented in Table 4. 
Table 2. Summary of mental health outcome measures of included studies

\begin{tabular}{|c|c|c|c|c|c|c|c|c|c|c|c|}
\hline \multirow[b]{2}{*}{ Study } & \multirow[b]{2}{*}{$\begin{array}{l}\text { Outcome } \\
\text { measure }\end{array}$} & \multicolumn{3}{|c|}{ Intervention } & \multirow[b]{2}{*}{ Post (SD) } & \multicolumn{3}{|c|}{ Control } & \multicolumn{3}{|c|}{ Both groups } \\
\hline & & $\begin{array}{l}\text { Intervention } \\
\text { name }\end{array}$ & $\mathbf{N}$ & Pre (SD) & & $\begin{array}{c}\text { Comparator } \\
\text { name }\end{array}$ & $\mathbf{N}$ & Pre & Post & $\mathrm{g}[95 \% \mathrm{Cl}]$ & Summary for MH measure \\
\hline \multirow[t]{2}{*}{$\begin{array}{l}\text { Bennett } \\
\text { (2017) }\end{array}$} & SDQ-P total & $\begin{array}{l}\text { Cognitive } \\
\text { Behavioural } \\
\text { therapy } \\
\text { guided self- } \\
\text { help }\end{array}$ & 17 & $22.41(3.50)$ & $18.78(1.77)$ & Waitlist control & 17 & 21.94(3.96) & $19.13(4.30)$ & $-0.10[-0.78,0.57]$ & $\begin{array}{l}\text { SDQ total scores demonstrated change } \\
\text { across time, and a medium/large effect size } \\
\text { was noted }(d=1.308,95 \% \text { Cl: } 0.568 \text { to } \\
2.049) \text {. There was no significant difference } \\
\text { between groups. }\end{array}$ \\
\hline & SDQ-P impact & & 17 & $5.94(2.36)$ & $5.07(1.93)$ & Waitlist control & 17 & $5.88(2.71)$ & $3.95(2.22)$ & $0.53[-0.16,1.21]$ & $\begin{array}{l}\text { The SDQ impact scale similarly } \\
\text { demonstrated a significant effect of time, but } \\
\text { no statistically significant difference between } \\
\text { groups. }\end{array}$ \\
\hline $\begin{array}{l}\text { Bufalini, et al. } \\
\text { (2009) }\end{array}$ & m-YPAS & $\begin{array}{l}\text { Interactive } \\
\text { Music therapy }\end{array}$ & 20 & $\begin{array}{l}\text { Measured at } \\
4 \text { time points } \\
\text { up until } \\
\text { procedure }\end{array}$ & $\begin{array}{l}\text { Measured at } 4 \\
\text { time points up } \\
\text { until procedure }\end{array}$ & $\begin{array}{c}\text { Treatment as } \\
\text { usual }\end{array}$ & 19 & $\begin{array}{l}\text { Measured } \\
\text { at } 4 \text { time } \\
\text { points up } \\
\text { until } \\
\text { procedure }\end{array}$ & $\begin{array}{l}\text { Measured at } \\
4 \text { time points } \\
\text { up until } \\
\text { procedure }\end{array}$ & $\begin{array}{l}\text { Not } \\
\text { reported/insufficient } \\
\text { data to calculate }\end{array}$ & $\begin{array}{l}\text { Significant decrease in procedural anxiety at } \\
\text { all } 4 \text { time points for intervention group } \\
\text { compared to controls. }\end{array}$ \\
\hline $\begin{array}{l}\text { Field et. al. } \\
\text { (1998) }\end{array}$ & STAI-C & $\begin{array}{l}\text { Massage } \\
\text { therapy }\end{array}$ & 8 & $\begin{array}{c}32.60 \text { (SD } \\
\text { NR) }\end{array}$ & 27.9 (SD NR) & $\begin{array}{c}\text { Progressive } \\
\text { muscle relaxation }\end{array}$ & 8 & $\begin{array}{l}30.70 \text { (SD } \\
\text { NR) }\end{array}$ & 28.8 (SD NR) & $\begin{array}{l}\text { Not } \\
\text { reported/insufficient } \\
\text { data to calculate }\end{array}$ & $\begin{array}{l}\text { Significant decrease in reported anxiety in } \\
\text { the older children and their parents } \\
\text { immediately after massage therapy. }\end{array}$ \\
\hline $\begin{array}{l}\text { Freedenberg } \\
\text { et.al. } \\
\text { (2017) }\end{array}$ & $\begin{array}{l}\text { HADS } \\
\text { (anxiety) }\end{array}$ & $\begin{array}{l}\text { Mindfulness- } \\
\text { Based Stress } \\
\text { Reduction }\end{array}$ & 26 & $10.5(4.0)$ & $9.9(4.0)$ & $\begin{array}{l}\text { Online video } \\
\text { support group }\end{array}$ & 20 & $7.1(6.2)$ & $6.8(5.3)$ & $0.66[0.06,1.26]$ & $\begin{array}{l}\text { Anxiety scores did not change significantly } \\
\text { from before to after the interventions. } \\
\text { The pre-post by group interaction revealed } \\
\text { that anxiety did not change differently in the } \\
\text { two groups. }\end{array}$ \\
\hline $\begin{array}{l}\text { Hains et.al. } \\
(2000)\end{array}$ & STAl-state & $\begin{array}{l}\text { Cognitive } \\
\text { behavioural } \\
\text { skills building }\end{array}$ & 8 & $39.00(7.45)$ & $33.75(6.43)$ & Waitlist control & 7 & $38.5(8.74)$ & 39.67 (10.93) & $\begin{array}{l}-0.63 \\
{[-1.68,0.41]}\end{array}$ & $\begin{array}{l}\text { Significant within group differences on state } \\
\text { anxiety. No statistically significant difference } \\
\text { between groups. }\end{array}$ \\
\hline $\begin{array}{l}\text { Hickman et.al. } \\
\text { (2015) }\end{array}$ & $\begin{array}{l}\text { BYI- } \\
\text { depression }\end{array}$ & $\begin{array}{l}\text { Group } \\
\text { cognitive } \\
\text { behavioural } \\
\text { therapy }\end{array}$ & 16 & $60(4.51)$ & $51.69(6.65)$ & Education group & 16 & $\begin{array}{l}57.56 \\
(3.35)\end{array}$ & $49.69(6.46)$ & $\begin{array}{l}0.30 \\
{[-0.40,0.99]}\end{array}$ & $\begin{array}{l}\text { No statistically significant difference was } \\
\text { found in post-intervention depression } \\
\text { controlling for baseline differences in } \\
\text { depression scores. }\end{array}$ \\
\hline $\begin{array}{l}\text { Jastrowski et. } \\
\text { al. } \\
\text { (2013) }\end{array}$ & STAI-C & $\begin{array}{l}\text { Mindfulness- } \\
\text { Based Stress } \\
\text { Reduction }\end{array}$ & 4 & Not reported & Not reported & Not reported & 2 & $\begin{array}{c}\text { Not } \\
\text { reported }\end{array}$ & Not reported & $\begin{array}{l}\text { Not } \\
\text { reported/insufficient } \\
\text { data to calculate }\end{array}$ & $\begin{array}{l}\text { Due to very high attrition levels, statistical } \\
\text { analyses could not be carried out and means } \\
\text { (SD) were not reported. }\end{array}$ \\
\hline
\end{tabular}




\begin{tabular}{|c|c|c|c|c|c|c|c|c|c|c|c|}
\hline \multirow[t]{2}{*}{$\begin{array}{l}\text { Liossi et. al. } \\
\text { (1998) }\end{array}$} & \multirow[t]{2}{*}{$\begin{array}{l}\text { Wong-Baker } \\
\text { FACES }\end{array}$} & \multirow[t]{2}{*}{ Hypnosis } & \multirow[t]{2}{*}{10} & \multirow[t]{2}{*}{$\begin{array}{l}4.5^{\mathrm{p}} \\
\text { (IQR not } \\
\text { reported) }\end{array}$} & \multirow[t]{2}{*}{$\begin{array}{l}0.5^{p} \\
\text { (IQR not } \\
\text { reported) }\end{array}$} & \multirow{2}{*}{$\begin{array}{l}\text { Cognitive } \\
\text { Behavioural } \\
\text { Therapy } \\
\text { Control }\end{array}$} & 10 & \multirow{2}{*}{$\begin{array}{l}5^{p}(\mathrm{IQR} \\
\text { not } \\
\text { reported }) \\
4.5^{p}(\mathrm{IQR} \\
\text { not } \\
\text { reported })\end{array}$} & \multirow{2}{*}{$\begin{array}{l}3.5^{\mathrm{p}} \\
\text { (IQR not } \\
\text { reported) } \\
\\
5.0^{\mathrm{p}} \\
\text { (IQR not } \\
\text { reported) }\end{array}$} & \multirow{2}{*}{$\begin{array}{l}\text { Not } \\
\text { reported/insufficient } \\
\text { data to calculate } \\
\text { Not } \\
\text { reported/insufficient } \\
\text { data to calculate }\end{array}$} & \multirow{2}{*}{$\begin{array}{l}\text { The individual comparisons between groups } \\
\text { were significant for anxiety scores. } \\
\text { The individual comparisons between groups } \\
\text { were significant for anxiety scores. }\end{array}$} \\
\hline & & & & & & & 10 & & & & \\
\hline \multirow[t]{2}{*}{ Scharff et. al. } & STAI-C & $\begin{array}{c}\text { Thermal } \\
\text { biofeedback } \\
\text { and cognitive } \\
\text { behavioural }\end{array}$ & 12 & $32.2(12.2)$ & Not reported & $\begin{array}{l}\text { Hand cooling } \\
\text { Biofeedback }\end{array}$ & 11 & $34.6(8.5)$ & Not reported & $\begin{array}{l}\text { Not } \\
\text { reported/insufficient } \\
\text { data to calculate }\end{array}$ & $\begin{array}{l}\text { Reported no significant difference in STAIC } \\
\text { score change between groups (pre, post) }\end{array}$ \\
\hline & STAI-C & $\begin{array}{l}\text { stress } \\
\text { management } \\
\text { training }\end{array}$ & 12 & $32.2(12.2)$ & Not reported & Waitlist control & 12 & $37.7(10.6)$ & Not reported & $\begin{array}{l}\text { Not } \\
\text { reported/insufficient } \\
\text { data to calculate }\end{array}$ & $\begin{array}{l}\text { Reported no significant difference in STAIC } \\
\text { score change between groups (pre, post) }\end{array}$ \\
\hline $\begin{array}{l}\text { Sharma et.al. } \\
\text { (2017) }\end{array}$ & STAI-state & $\begin{array}{l}\text { Transdiagnost } \\
\text { ic group CBT }\end{array}$ & 32 & $41.00(9.39)$ & $29.16(5.97)$ & $\begin{array}{l}\text { Treatment as } \\
\text { usual }\end{array}$ & 31 & $42.42(8.71)$ & $40.13(9.02)$ & $\begin{array}{l}-1.42 \\
{[-1.98,-0.86]}\end{array}$ & $\begin{array}{l}\text { Participants in the TCBT condition showed } \\
\text { significant improvement in state anxiety, } \\
\text { while no significant change in state anxiety } \\
\text { was observed for participants in the TAU } \\
\text { condition. }\end{array}$ \\
\hline $\begin{array}{l}\text { Yetwin } \\
\text { (2011) }\end{array}$ & CASI & $\begin{array}{c}\text { HRV } \\
\text { Biofeedback }\end{array}$ & 9 & $29.78(7.48)$ & $28.67(7.26)$ & Waitlist control & 10 & $\begin{array}{l}31.90 \\
(8.17)\end{array}$ & $28.60(7.55)$ & $0.01[-0.89,0.91]$ & $\begin{array}{l}\text { No significant between group difference on } \\
\text { anxiety sensitivity. }\end{array}$ \\
\hline $\begin{array}{l}\text { Zhang et.al. } \\
\text { (2019) }\end{array}$ & $\begin{array}{l}\text { DASS } \\
\text { (anxiety) }\end{array}$ & CBT & 53 & $11.53(7.52)$ & $5.83(3.07)$ & $\begin{array}{l}\text { Treatment as } \\
\text { usual }\end{array}$ & 53 & $9.49(4.61)$ & $8.66(4.92)$ & $\begin{array}{l}-0.69 \\
{[-1.08,-0.29]}\end{array}$ & $\begin{array}{l}\text { Participants in the CBT group showed } \\
\text { significant improvement in anxiety, while no } \\
\text { significant change in anxiety was observed } \\
\text { for participants in the TAU condition. }\end{array}$ \\
\hline
\end{tabular}


The two studies with the largest sample sizes and a relatively low risk of bias were Sharma and colleagues (2017) and Zhang and colleagues (2019). Zhang and colleagues (2019) showed that 5 sessions of CBT in young people with cancer significantly improved symptoms of anxiety compared to treatment as usual ( $n=106$ in the completers analysis). In Sharma et al. (2017) ( $n=63)$ a transdiagnostic CBT group intervention significantly improved symptoms of anxiety in young people with a headache disorder compared to treatment as usual.

Evidence for the effect of CBT on anxiety in children with a long term physical health condition and any comparator was investigated further by meta-analysing outcomes for three studies: Hains and colleagues (2000), Sharma and colleagues (2017) and Zhang and colleagues (2019). We did not include the study by Bennett and colleagues (2017), as the only mental health measure where participants had above threshold symptoms in both arms at baseline was the Strengths and Difficulties Questionnaire. Although the Strengths and Difficulties Questionnaire covers anxiety, it is a summary measure of emotional and behaviour symptoms and therefore was deemed too different from the DASS (anxiety) and STAI-state used in the included studies, which specifically measure symptoms of anxiety. A large effect size in favour of brief CBT was found $(\mathrm{g}=-0.95, \mathrm{Cl}-1.49$ to $-.041 ; \mathrm{p}<0.01)$ with non-significant moderate-substantial heterogeneity $\left(I^{2}=58 \% ; p=0.09\right)$. The interventions included young people with three different long term physical health conditions: paediatric cancer, headache and type I diabetes and two different control groups (pharmacotherapy as part of treatment as usual for two studies and waitlist control for ones). The results of the meta-analysis are summarised in a forest plot (see Figure 4). 


\begin{tabular}{|c|c|c|c|c|c|c|}
\hline \multirow[b]{2}{*}{ Study or Subgroup } & \multicolumn{3}{|c|}{ Experimental } & \multicolumn{3}{|c|}{ Control } \\
\hline & Mean & SD & Total & Mean & SD & \\
\hline Hains (2000) & 33.75 & 6.43 & 8 & 39.67 & 10.93 & \\
\hline Sha & 29.16 & 5.97 & 32 & 40.13 & 9.02 & \\
\hline Zhang (2019) & 5.83 & 3.07 & 53 & 8.66 & 4.92 & \\
\hline Total $(95 \% \mathrm{Cl})$ & & & 93 & & & \\
\hline \multicolumn{7}{|c|}{$\begin{array}{l}\text { Heterogeneity: } \operatorname{Tau}^{2}=0.13 ; \mathrm{Chi}^{2}=4.73, \mathrm{df}=2(\mathrm{P}=0.09) ;\left.\right|^{2}=5 \\
\text { Test for overall effect: } \mathrm{Z}=3.43(\mathrm{P}=0.0006)\end{array}$} \\
\hline \multicolumn{7}{|l|}{ Risk of bias legend } \\
\hline \multicolumn{7}{|c|}{ (A) Random sequence generation (selection bias) } \\
\hline \multicolumn{7}{|c|}{ (B) Allocation concealment (selection bias) } \\
\hline \multicolumn{7}{|c|}{ (C) Blinding of participants and personnel (performance bia } \\
\hline \multirow{2}{*}{\multicolumn{7}{|c|}{ (D) Blinding of outcome assessment (detection bias) }} \\
\hline & & & & & & \\
\hline (F) $\mathrm{Se}$ & & & & & & \\
\hline
\end{tabular}

Std. Mean Difference

Std. Mean Difference

Risk of Bias

Figure 4. Forest plot of comparison: CBT vs any comparator, outcome: anxiety post-intervention 
Mindfulness-based stress reduction interventions in one case showed no effect on anxiety or depression (Freedenberg et al., 2017) and in the other (Jastrowski Mano et al., 2013) considerable attrition meant effect sizes could not be calculated. Both biofeedback interventions found no significant between group difference on anxiety scores (Scharff et al., 2002; Yetwin, 2011). Massage therapy (Field et al., 1998), hypnosis (Liossi \& Hatira, 1999) and music therapy (Bufalini, 2009) all reported significant differences between groups for anxiety scores. However, these studies had a higher risk of bias, small sample sizes and lacked replication for the particular intervention type. There was insufficient evidence to comment on whether brief interventions have an effect on physical health outcomes or quality of life. Similarly, a lack of evidence precluded conclusions on possible moderators of efficacy. 
Table 3. Summary of physical health and other outcomes of included studies

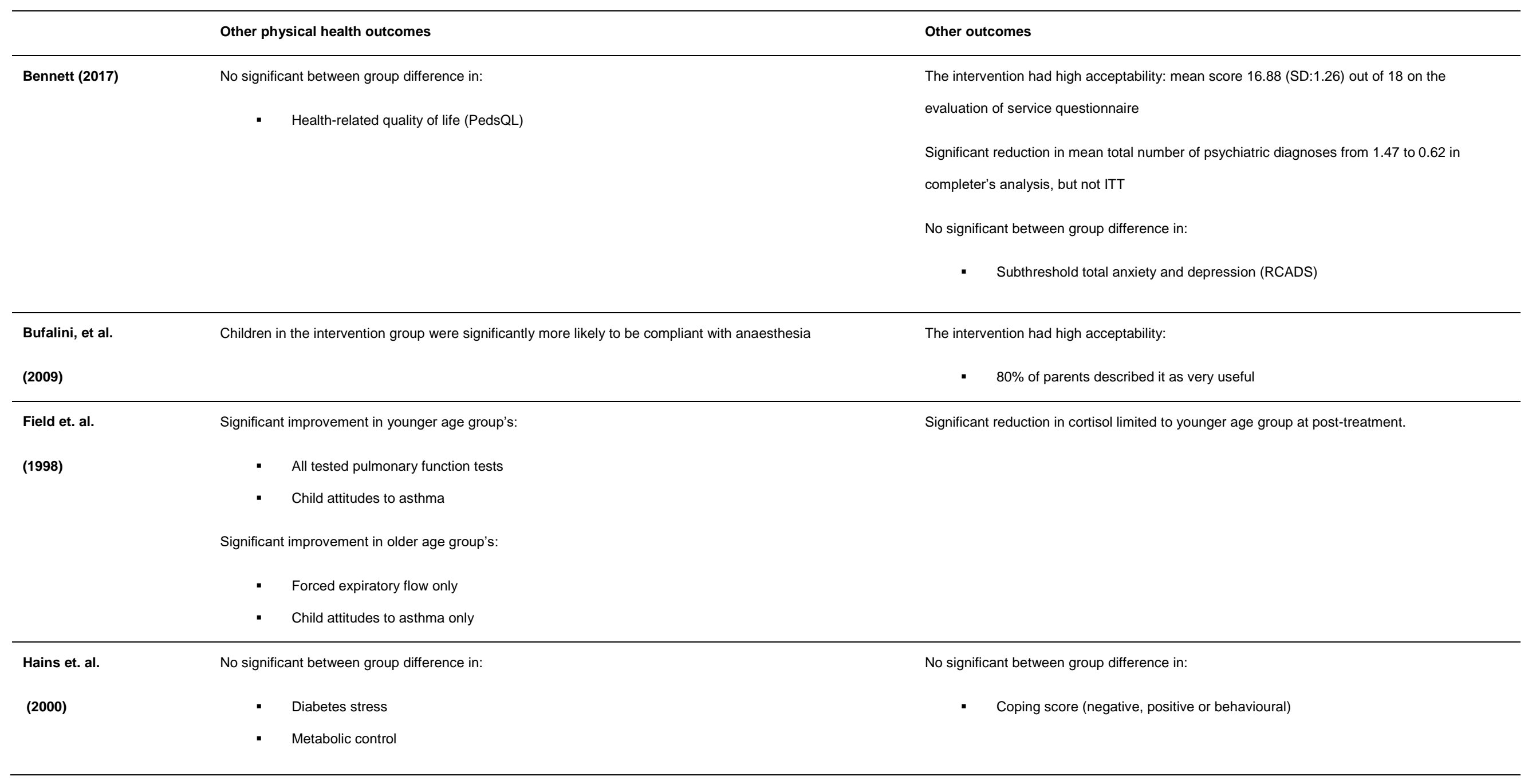




\section{Hickman et.al.}

(2015)

- Significant within, but not between group difference in self-reported reduction of headache-related disability

- Significant between group difference in self-reported increase in beliefs about teen's ability to lead a healthy lifestyle in intervention group but not in comparison group.

No significant change in parent-reported reduction in parent perception of pain interference
The intervention had high acceptability:

- $94 \%$ rated the COPE-HEP intervention as helpful

- $100 \%$ of parents and adolescents would recommend the intervention to a friend

- $100 \%$ adolescents completed all 7 sessions, although at varying times
Freedenberg et.al. Significant within, but not between group difference in illness-related stress

(2017)

Significant within, but not between, group differences in secondary coping
Predictors of increased reduction in anxiety/depression symptoms:

- Higher baseline levels of anxiety/depression respectively

- Greater use of coping of coping skills
Jastrowski et. al.

(2013)

\section{Liossi et. al.}

(1998)
Due to very high attrition levels, statistical analyses could not be carried out and means (SD) were not reported.

Significant reduction in self-reported pain:

- Between Hypnosis and control

- Between CBT and control
Due to very high attrition levels, statistical analyses could not be carried out and means (SD) were not reported.

No significant between group difference (CBT vs Hypnosis) in correlations between hypnotisability and clinical outcomes

No significant difference between hypnosis and CBT for self-reported pain

\section{Scharff et.al.}

(1999)
Significant between group difference in biofeedback groups compared to WLC in reduced:

None.
- headache frequency

- headache index

Within group gains maintained at 3- and 6- month follow-up

No significant between group difference in headache variables at 3- or 6- months follow-up between

biofeedback groups 


\section{Sharma et.al.}

(2017)

Significant within and between group difference in:

- Self-reported reduction of headache impact on daily life (in both groups from pre- to post-, but significantly greater for those receiving TCBT than for those in the TAU condition)
Significant within and between group difference in:

- Clinician-rated improvement in young person's global functioning (in both groups from pre- to post-, but significantly greater for those receiving TCBT than for those in the TAU condition)

\section{Yetwin}

Significant between group difference in:

(2011)

- $\quad$ Self-reported reduction in pain intensity

- Self-reported reduction in 'current pain' (PPQ)

- $\quad$ Self-reported increase in school functioning (PedsQL)

No significant between group difference in:

- Sleep quality

- $\quad$ Self-reported 'worst pain' (PPQ)

- Self-reported 'Total', 'physical', 'emotional' and 'social' functioning (PedsQL)
No significant between group difference in:

- Subthreshold depression

\section{Zhang et.al.}

(2019)
None
Significant between group difference in:

- Reduction of subthreshold symptoms of low mood and stress

- Increase in psychological resilience

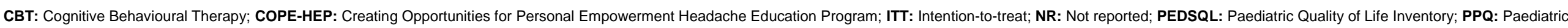
Pain Questionnaire; RCADS: Revised Children's Anxiety and Depression Scale; SD: Standard Deviation; TAU: treatment as usual; TCBT: Transdiagnostic cognitive behavioural therapy; WLC: Waitlist control; 
Table 4. Summary of Findings table

Brief interventions compared to any comparator for psychiatric disorders in children with long term conditions

Patient or population: Children and adolescents with psychiatric disorders and long term conditions

Settings:

Intervention: brief therapy

Comparison: any comparator

\begin{tabular}{|c|c|c|c|c|c|}
\hline Outcomes & Anticipate & d absolute effects $(95 \% \mathrm{Cl})$ & $\begin{array}{l}\text { No of } \\
\text { Participants } \\
\text { (studies) }\end{array}$ & $\begin{array}{l}\text { Quality of the } \\
\text { evidence } \\
\text { (GRADE) }\end{array}$ & Comments \\
\hline Anxiety post-intervention: CBT vs any comparator & & $\begin{array}{l}\text { The mean anxiety post-intervention: cbt vs any } \\
\text { comparator in the intervention groups was } \\
0.95 \text { standard deviations lower } \\
\text { (1.49 to } 0.41 \text { lower) }\end{array}$ & $\begin{array}{l}184 \\
\text { (3 studies) }\end{array}$ & $\begin{array}{l}\oplus \oplus \oplus \ominus \\
\text { moderate }^{1,2,3}\end{array}$ & SMD $-0.95(-1.49$ to -0.41$)$ \\
\hline Anxiety post-intervention: MBSR vs any comparator & $\begin{array}{l}\text { See } \\
\text { comment }\end{array}$ & See comment & $\begin{array}{l}52 \\
\text { (2 studies) }\end{array}$ & $\underset{\text { low }^{4}}{\oplus \oplus \Theta}$ & $\begin{array}{l}\text { No significant between group } \\
\text { difference. }\end{array}$ \\
\hline Anxiety post-intervention: Biofeedback vs any comparator & $\begin{array}{l}\text { See } \\
\text { comment }\end{array}$ & See comment & $\begin{array}{l}43 \\
\text { (2 studies) }\end{array}$ & $\begin{array}{l}\oplus \ominus \Theta \Theta \\
\text { very low }\end{array}$ & $\begin{array}{l}\text { No significant between group } \\
\text { difference. }\end{array}$ \\
\hline $\begin{array}{l}\text { Anxiety post-intervention: Other brief therapy (music therapy, } \\
\text { massage therapy and hypnosis) vs any comparator }\end{array}$ & $\begin{array}{l}\text { See } \\
\text { comment }\end{array}$ & See comment & $\begin{array}{l}75 \\
\text { (3 studies) }\end{array}$ & $\begin{array}{l}\oplus \Theta \Theta \Theta \\
\text { very low }\end{array}$ & $\begin{array}{l}\text { Significant reduction in anxiety } \\
\text { in intervention groups. }\end{array}$ \\
\hline \multicolumn{6}{|c|}{ Cl: Confidence interval ; SMD: Standardised Mean Difference ; MBSR: Mindfulness-based stress reduction; CBT: Cognitive Behavioural Therapy } \\
\hline \multicolumn{6}{|c|}{$\begin{array}{l}\text { GRADE Working Group grades of evidence } \\
\text { High quality: Further research is very unlikely to change our confidence in the estimate of effect. } \\
\text { Moderate quality: Further research is likely to have an important impact on our confidence in the estimate of effect and may change the estimate. } \\
\text { Low quality: Further research is very likely to have an important impact on our confidence in the estimate of effect and is likely to change the estimate. } \\
\text { Very low quality: We are very uncertain about the estimate. }\end{array}$} \\
\hline \multicolumn{6}{|c|}{$\begin{array}{l}1 \text { Unclear or high risk of bias in a number of domains } \\
{ }^{2} \text { Small sample sizes in included studies } \\
{ }^{3} \text { Large pooled effect size } \\
{ }^{4} \text { Very high attrition in one study. } \\
{ }^{5} \text { High or unclear risk of bias in most domains across studies. } \\
{ }^{6} \text { No replication of therapy type by independent group (e.g. only one study looking at massage therapy) }\end{array}$} \\
\hline
\end{tabular}




\section{Discussion}

\section{Summary of main results}

This review and meta-analysis provide preliminary evidence that brief interventions based on cognitive behavioural principles may benefit young people with a long term physical health conditions who also present with elevated symptoms of anxiety.

There was insufficient evidence to assess whether this held true for depression, disruptive behaviour/oppositional defiant disorder, other psychiatric disorders, health-related quality of life or physical health. Similarly there was insufficient evidence to establish possible moderators of treatment efficacy or acceptability.

Music therapy, hypnosis and massage therapy showed some promise, but studies looking at these interventions displayed high risk of bias, small sample sizes and lacked replication by independent research groups, so more research is needed in this area. However, we also recognise that it may be difficult to maintain low bias in all areas, particularly in blinding participants, for these types of intervention. At present there is insufficient evidence to recommend mindfulness-based stress reduction, biofeedback, hypnosis, music therapy or massage therapy as brief interventions for this population.

\section{Limitations}

The findings from the meta-analysis must be interpreted with caution due to high risk of bias in 6 domains across the three included studies, small number of included studies and sample sizes (pooled intervention arm: $n=93$ and pooled comparator arm: $n=91$ ) and differences in chronic conditions (cancer, headache and type 1 diabetes) and control groups (pharmacotherapy as part of treatment as usual for two studies and waitlist control for one).

One of the studies included in this review was conducted by four of the review authors (SB, AC, IH, RS; (Bennett, 2017), which may be a potential source of bias. However, data 
extraction and risk of bias were conducted independently by two authors who had no involvement with the original study. Two of the included studies were unpublished and so did not undergo peer review. We could not formally assess publication bias due to the limited number of studies in the meta-analysis and as a result cannot exclude the possibility of publication bias. However, we did run pre-defined searches in a number of databases and searched grey literature to try and minimise the risk. It is possible that the search terms we selected biased the findings in favour of cognitive behavioural therapy. All studies used selfreport measures for the outcomes of interest and were therefore at risk of 'halo effects' and other response biases.

\section{Agreements and disagreements with other studies or reviews}

In keeping with previous reviews in the area (Bennett et al., 2015; Moore et al., 2019; Thabrew et al., 2018; Thabrew et al., 2017) we found tentative evidence for CBT-based interventions. Our findings diverged from those found in a review by Thabrew and colleagues (2018) who conducted a subgroup analysis to establish the effects of 'brief' CBT for anxiety in young people with a long term physical health condition. In their meta-analysis a non-significant between group difference was found between brief psychological treatments ( $<6$ hours or $<6$ sessions) versus any comparator at post-treatment. The difference is likely due to methodological differences in the reviews. For example, we only included studies in which symptoms of anxiety were elevated above a clinical cut-off on a validated measure. We also did not synthesise different types of psychological therapies in the same meta-analysis (e.g. CBT and family therapy). Moreover, we considered group treatment as brief (if it met our criteria of $\leq 10$ sessions with $\leq 6$ hours of therapist contact per patient). This for example, led to one study (Sharma et al., 2017), being classed as 'longer therapy' in Thabrew and colleagues (2018), but as 'brief' in our review. Future work should look towards obtaining a consensus as to what comprises a 'brief' intervention and how it applies to group treatment. Finally, we found an additional RCT published since their review: Zhang and colleagues (2019). 
Compared to the evidence synthesis by Moore et.al. (2019) who found tentative evidence for CBT for depression, we only found one study using brief CBT, in which symptoms of depression were above threshold and it showed no between-group differences when correcting for baseline differences in depression (Hickman et al., 2015). The main reason for excluding many of the studies from our review were either to do with the interventions being high-intensity (e.g. several of the inflammatory bowel disease interventions) or due to the sample means being below threshold for a psychiatric disorder. Again, the main divergence here were to do with methodological differences and the scope of the reviews.

In keeping with a previous review by Bennett et. al (2015), study authors reported having to make allowances for young people and families living with long term physical health conditions; for example cancellations were common (Hickman et al., 2015). In certain studies, telephone therapy was used to make appointments more accessible (Bennett, 2017). Others reflected post-hoc that including more telephone sessions, might increase feasibility (Hickman et al., 2015).

Implications for practice and future research

The findings from this review suggest that brief CBT may be an effective way to increase access to treatment for elevated symptoms of anxiety in young people with a long term physical health condition. These could be delivered in a group or individual format. Brief CBT treatments for anxiety may form part of a 'stepped care approach' in existing paediatric services.

Trials of brief parenting interventions for disruptive behaviour and brief interventions for depression need to be carried out, as these have been shown to be effective in young people without a long term physical health condition (Bennett et al., 2019) and/or in a highintensity format in children with a long term physical health condition (Moore et al., 2019). A 
greater number of appropriately powered trials with mental health as the primary outcome are also required. This review focused on children that were above threshold for a mental health disorder, but there is an important public health impact of improving mental health in those with long term physical health conditions even if they are subthreshold, so additional syntheses of those studies would be useful. Larger, high quality trials targeting elevated anxiety symptoms need to replicate the preliminary findings in this review and include longer term follow-ups. As a number of evidence-based brief treatments for anxiety for children without a long term physical health condition already exist, these could be adapted and tested in future trials. Trials evaluating stepped care approaches for young people with a long term physical health condition need to be conducted to establish who benefits from brief therapy, who may need to be 'stepped up' and how services can best be organised to meet rising demands.

At a time when child and adolescent mental health services have been publicly described as "a car crash waiting to happen"(Doward, 2016), funding and research into brief CBT interventions may provide some of the increased access that is so urgently required.

Funding: This work was supported by the Beryl Alexander Charity and Great Ormond Street Hospital Children's Charity (Project grant number: 16HN11 and PhD grant number: 18PP19).

Acknowledgements: All research at Great Ormond Street Hospital NHS Foundation Trust and UCL Great Ormond Street Institute of Child Health is made possible by the NIHR Great Ormond Street Hospital Biomedical Research Centre. The views expressed are those of the author(s) and not necessarily those of the NHS, the NIHR or the Department of Health. 
Conflicts of interest: the authors have no competing interests to report.

\section{Tables}

1. Characteristics of included studies

2. Summary of mental health outcome measures of included studies

3. Summary of physical health and other outcomes of included studies

4. Summary of Findings table

\section{Figures}

1. PRISMA flow diagram of included studies in the review

2. Risk of bias summary: review authors' judgements about each risk of bias item for each included study

3. Risk of bias graph: review authors' judgements about each risk of bias item presented as percentages across all included studies

4. Forest plot of comparison: CBT vs any comparator, outcome: anxiety postintervention 


\section{References}

Almane, D. N., Jones, J. E., McMillan, T., Stafstrom, C. E., Hsu, D. A., Seidenberg, M., . . Oyegbile, T. O. (2019). The Timing, Nature, and Range of Neurobehavioral Comorbidities in Juvenile Myoclonic Epilepsy. Pediatr Neurol. doi:10.1016/j.pediatrneurol.2019.03.011

Bennett. (2017). Mental Health of Children and Young People with Neurological Conditions. Bennett, Cuijpers, P., Ebert, D. D., McKenzie Smith, M., Coughtrey, A. E., Heyman, I., . . . Shafran, R. (2019). Practitioner Review: Unguided and guided self-help interventions for common mental health disorders in children and adolescents: a systematic review and meta-analysis. J Child Psychol Psychiatry. doi:10.1111/jcpp.13010

Bennett, S., Shafran, R., Coughtrey, A., Walker, S., \& Heyman, I. (2015). Psychological interventions for mental health disorders in children with chronic physical illness: a systematic review. Arch Dis Child, 100(4), 308-316. doi:10.1136/archdischild-2014307474

Birnie, K. A., Noel, M., Chambers, C. T., Uman, L. S., \& Parker, J. A. (2018). Psychological interventions for needle-related procedural pain and distress in children and adolescents. Cochrane Database Syst Rev, 10, CD005179. doi:10.1002/14651858.CD005179.pub4

Brooks, F., Magnusson, J., Klemera, E., Spencer, N., \& Morgan, A. (2011). Health Behaviour in School-aged Children (HBSC): World Health Organization Collaborative Cross National Study. . Retrieved from

Bufalini. (2009). Role of interactive music in oncological patients undergoing painful procedures.

Cape, J., \& Kendall, T. (2011). Generalised Anxiety Disorder in adults. Retrieved from https://www.ncbi.nIm.nih.gov/books/NBK83456/

Chun, Y. H., Han, K., Park, Y. G., Yoon, J. S., Kim, H. H., Kim, J. T., \& Jeong, D. C. (2015).

Examining impacts of allergic diseases on psychological problems and tobacco use in Korean adolescents: the 2008-2011 Korean National Health and Nutrition Examination Survey. PLoS One, 10(4), e0125172. doi:10.1371/journal.pone.0125172

Children's Commissioner. (2016). Lightning Review: Access to Child and Adolescent Mental Health Services. Retrieved from https://www.childrenscommissioner.gov.uk/wpcontent/uploads/2017/06/Childrens-Commissioners-Mental-Health-LightningReview.pdf

Conviser, J. H., Fisher, S. D., \& McColley, S. A. (2018). Are children with chronic illnesses requiring dietary therapy at risk for disordered eating or eating disorders? A systematic review. Int J Eat Disord, 51(3), 187-213. doi:10.1002/eat.22831

Cousino, M. K., \& Hazen, R. A. (2013). Parenting stress among caregivers of children with chronic illness: a systematic review. J Pediatr Psychol, 38(8), 809-828. doi:10.1093/jpepsy/jst049

Creswell, C., Violato, M., Fairbanks, H., White, E., Parkinson, M., Abitabile, G., . . Cooper, P. J. (2017). Clinical outcomes and cost-effectiveness of brief guided parent-delivered cognitive behavioural therapy and solution-focused brief therapy for treatment of childhood anxiety disorders: a randomised controlled trial. The Lancet Psychiatry, 4(7), 529-539.

Ding, T., Hall, A., Jacobs, K., \& David, J. (2008). Psychological functioning of children and adolescents with juvenile idiopathic arthritis is related to physical disability but not 
to disease status. Rheumatology (Oxford), 47(5), 660-664.

doi:10.1093/rheumatology/ken095

Doward, J. (2016). Act on children's mental ill health or risk national crisis, warns expert. The Guardian. Retrieved from https://www.theguardian.com/society/2016/oct/01/fundnhs-child-mental-health-services-to-avoid-crisis

Edbrooke-Childs, J., Calderon, A., Wolpert, M., \& Fonagy, P. (2015). CYP IAPT Rapid Internal Audit. Retrieved from https://www.ucl.ac.uk/evidence-based-practiceunit/sites/evidence-based-practiceunit/files/pub and resources project reports rapid internal audit 2015.pdf

Field, T., Henteleff, T., Hernandez-Reif, M., Martinez, E., Mavunda, K., Kubn, C., \& Schanberg, S. (1998). Children with asthma have improved pulmonary functions after massage therapy. Journal of Pediatrics, 132(5), 854-858. doi:Doi 10.1016/S0022-3476(98)70317-8

Fisher, E., Law, E., Palermo, T. M., \& Eccleston, C. (2015). Psychological therapies (remotely delivered) for the management of chronic and recurrent pain in children and adolescents. Cochrane Database Syst. Rev.(3), CD011118. doi:10.1002/14651858.CD011118.pub2

Freedenberg, V. A., Hinds, P. S., \& Friedmann, E. (2017). Mindfulness-Based Stress Reduction and Group Support Decrease Stress in Adolescents with Cardiac Diagnoses: A Randomized Two-Group Study. Pediatr Cardiol, 38(7), 1415-1425. doi:10.1007/s00246-017-1679-5

Hains. (2000). A stress management intervention for youth with type I diabetes.

Hickman, C., Jacobson, D., \& Melnyk, B. M. (2015). Randomized controlled trial of the acceptability, feasibility, and preliminary effects of a cognitive behavioral skills building intervention in adolescents with chronic daily headaches: a pilot study. $J$ Pediatr Health Care, 29(1), 5-16. doi:10.1016/j.pedhc.2014.05.001

Higgins, J., Thomas, J., Chandler, J., Cumpston, M., Li, T., Page, M., \& Welch, V. e. (2019). Cochrane Handbook for Systematic Reviews of Interventions version 6.0. Retrieved from www.training.cochrane.org/handbook

Higgins, J. P. T., Altman, D. G., Gotzsche, P. C., Juni, P., Moher, D., Oxman, A. D., . . Cochrane Statistical Methods, G. (2011). The Cochrane Collaboration's tool for assessing risk of bias in randomised trials. BMJ, 343(oct18 2), d5928-d5928. doi:10.1136/bmj.d5928

Jastrowski Mano, K. E., Salamon, K. S., Hainsworth, K. R., Anderson Khan, K. J., Ladwig, R. J., Davies, W. H., \& Weisman, S. J. (2013). A randomized, controlled pilot study of mindfulness-based stress reduction for pediatric chronic pain. Altern Ther Health Med, 19(6), 8-14. Retrieved from https://www.ncbi.nlm.nih.gov/pubmed/24254033

Johnson, E. K., Jones, J. E., Seidenberg, M., \& Hermann, B. P. (2004). The relative impact of anxiety, depression, and clinical seizure features on health-related quality of life in epilepsy. Epilepsia, 45(5), 544-550. doi:10.1111/j.0013-9580.2004.47003.x

Kellett, S., Webb, K., Wilkinson, N., Bliss, P., Ayers, T., \& Hardy, G. (2016). Developing Services for Patients with Depression or Anxiety in the Context of Long-term Physical Health Conditions and Medically Unexplained Symptoms: Evaluation of an IAPT Pathfinder Site. Behav Cogn Psychother, 44(5), 553-567. doi:10.1017/S1352465816000114

Khanna, A. D., Duca, L. M., Kay, J. D., Shore, J., Kelly, S. L., \& Crume, T. (2019). Prevalence of Mental Illness in Adolescents and Adults With Congenital Heart Disease from the 
Colorado Congenital Heart Defect Surveillance System. Am J Cardiol, 124(4), 618626. doi:10.1016/j.amjcard.2019.05.023

Liossi, C., \& Hatira, P. (1999). Clinical hypnosis versus cognitive behavioral training for pain management with pediatric cancer patients undergoing bone marrow aspirations. Int J Clin Exp Hypn, 47(2), 104-116. doi:10.1080/00207149908410025

Moher, D., Liberati, A., Tetzlaff, J., Altman, D. G., \& Group, P. (2009). Preferred reporting items for systematic reviews and meta-analyses: the PRISMA statement. BMJ, 339, b2535. doi:10.1136/bmj.b2535

Moore, Nunns, M., Shaw, L., Rogers, M., Walker, E., Ford, T., ... Thompson Coon, J. (2019). Interventions to improve the mental health of children and young people with longterm physical conditions: linked evidence syntheses. Health Technol Assess, 23(22), 1-164. doi:10.3310/hta23220

NICE. (2013a). Antisocial behaviour and conduct disorders in children and young people: recognition and management. In: National Institute of Clinical Excellence.

NICE. (2013b). Social anxiety disorder: recognition, assessment and treatment. In.

NICE. (2015). Challenging behaviour and learning disabilities: prevention and interventions for people with learning disabilities whose behaviour challenges. In.

NICE. (2019). Depression in children and young people: identification and management. In. O'Neill, L. P., \& Murray, L. E. (2016). Anxiety and depression symptomatology in adult siblings of individuals with different developmental disability diagnoses. Res Dev Disabil, 51-52, 116-125. doi:10.1016/j.ridd.2015.12.017

Olusunmade, M., Qadir, T., Akyar, S., Farid, A., \& Aggarwal, R. (2019). Incremental hospital utilization and mortality associated with co-morbid depression in pediatric hospitalizations. J Affect Disord, 251, 270-273. doi:10.1016/j.jad.2019.03.073

Owens, P. L., Hoagwood, K., Horwitz, S. M., Leaf, P. J., Poduska, J. M., Kellam, S. G., \& Ialongo, N. S. (2002). Barriers to children's mental health services. J Am Acad Child Adolesc Psychiatry, 41(6), 731-738. doi:10.1097/00004583-200206000-00013

Pinquart, M. (2018). Posttraumatic Stress Symptoms and Disorders in Children and Adolescents with Chronic Physical Illnesses: a Meta-Analysis. Journal of Child \& Adolescent Trauma, 13(1), 1-10. doi:10.1007/s40653-018-0222-z

Pinquart, M., \& Shen, Y. (2011a). Anxiety in children and adolescents with chronic physical illnesses: a meta-analysis. Acta Paediatr, 100(8), 1069-1076. doi:10.1111/j.16512227.2011.02223.x

Pinquart, M., \& Shen, Y. (2011b). Behavior problems in children and adolescents with chronic physical illness: a meta-analysis. J Pediatr Psychol, 36(9), 1003-1016. doi:10.1093/jpepsy/jsr042

Pinquart, M., \& Shen, Y. (2011c). Depressive symptoms in children and adolescents with chronic physical illness: an updated meta-analysis. J Pediatr Psychol, 36(4), 375-384. doi:10.1093/jpepsy/jsq104

Quilter, M. C., Hiraki, L. T., \& Korczak, D. J. (2019). Depressive and anxiety symptom prevalence in childhood-onset systemic lupus erythematosus: A systematic review. Lupus, 28(7), 878-887. doi:10.1177/0961203319853621

Rodgers, M., Asaria, M., Walker, S., McMillan, D., Lucock, M., Harden, M., . . Eastwood, A. (2012). The clinical effectiveness and cost-effectiveness of low-intensity psychological interventions for the secondary prevention of relapse after depression: a systematic review. Health Technology Assessment, 16(28), 1-+. doi:10.3310/hta16280 
Scharff, L., Marcus, D. A., \& Masek, B. J. (2002). A controlled study of minimal-contact thermal biofeedback treatment in children with migraine. J Pediatr Psychol, 27(2), 109-119. doi:10.1093/jpepsy/27.2.109

Schünemann, H., Brożek, J., Guyatt, G., \& Oxman, A. (2013). Handbook for grading the quality of evidence and the strength of recommendations using the GRADE approach. Retrieved from gdt.guidelinedevelopment.org/app/handbook/handbook.html

Sharma, P., Mehta, M., \& Sagar, R. (2017). Efficacy of transdiagnostic cognitive-behavioral group therapy for anxiety disorders and headache in adolescents. $J$ Anxiety Disord, 46, 78-84. doi:10.1016/j.janxdis.2016.11.001

Sharpe, D., \& Rossiter, L. (2002). Siblings of children with a chronic illness: a meta-analysis. J Pediatr Psychol, 27(8), 699-710. doi:10.1093/jpepsy/27.8.699

Sildorf, S. M., Breinegaard, N., Lindkvist, E. B., Tolstrup, J. S., Boisen, K. A., Teilmann, G. K., . . . Svensson, J. (2018). Poor Metabolic Control in Children and Adolescents With Type 1 Diabetes and Psychiatric Comorbidity. Diabetes Care, 41(11), 2289-2296. doi:10.2337/dc18-0609

Stallard, P. (2017). Low-intensity interventions for anxiety disorders. The Lancet Psychiatry, 4(7), 508-509.

Thabrew, H., Stasiak, K., Hetrick, S. E., Donkin, L., Huss, J. H., Highlander, A., . . Merry, S. N. (2018). Psychological therapies for anxiety and depression in children and adolescents with long-term physical conditions. Cochrane Database Syst. Rev., 12, CD012488. doi:10.1002/14651858.CD012488.pub2

Thabrew, H., Stasiak, K., Hetrick, S. E., Wong, S., Huss, J. H., \& Merry, S. N. (2017). eHealth interventions for anxiety and depression in children and adolescents with long-term physical conditions. Cochrane Database of Systematic Reviews, 2017 (1) (no pagination)(CD012489).

Van Cleave, J., Gortmaker, S. L., \& Perrin, J. M. (2010). Dynamics of Obesity and Chronic Health Conditions Among Children and Youth. Jama-Journal of the American Medical Association, 303(7), 623-630. doi:10.1001/jama.2010.104

van der Lee, J. H., Mokkink, L. B., Grootenhuis, M. A., Heymans, H. S., \& Offringa, M. (2007). Definitions and measurement of chronic health conditions in childhood: a systematic review. JAMA, 297(24), 2741-2751. doi:10.1001/jama.297.24.2741

Weisz, J. R., Kuppens, S., Ng, M. Y., Eckshtain, D., Ugueto, A. M., Vaughn-Coaxum, R., . . Fordwood, S. R. (2017). What five decades of research tells us about the effects of youth psychological therapy: A multilevel meta-analysis and implications for science and practice. The American psychologist, 72(2), 79-117.

Welch, A., Shafran, R., Heyman, I., Coughtrey, A., \& Bennett, S. (2018). Usual care for mental health problems in children with epilepsy: A cohort study. F1000Research, 7, 1907. doi:10.12688/f1000research.15492.1

Yetwin. (2011). Heart Rate Variability Biofeedback Therapy for Children and Adolescents with Chronic Pain.

Zhang, P., Mo, L., Torres, J., \& Huang, X. (2019). Effects of cognitive behavioral therapy on psychological adjustment in Chinese pediatric cancer patients receiving chemotherapy: A randomized trial. Medicine (Baltimore), 98(27), e16319. doi:10.1097/MD.0000000000016319

Zima, B. T., Rodean, J., Hall, M., Bardach, N. S., Coker, T. R., \& Berry, J. G. (2016). Psychiatric Disorders and Trends in Resource Use in Pediatric Hospitals. Pediatrics, 138(5). doi:10.1542/peds.2016-0909 